\title{
Growth of Solutions with Positive Initial Energy to Systems of Nonlinear Wave Equations with Damping and Source Terms
}

\author{
Erhan Pişkin \\ Department of Mathematics, Dicle University, 21280 Diyarbakir, Turkey \\ Correspondence should be addressed to Erhan Pişkin; episkin@dicle.edu.tr
}

Received 27 November 2014; Accepted 15 February 2015

Academic Editor: Ivan Avramidi

Copyright (C) 2015 Erhan Pişkin. This is an open access article distributed under the Creative Commons Attribution License, which permits unrestricted use, distribution, and reproduction in any medium, provided the original work is properly cited.

We consider initial-boundary conditions for coupled nonlinear wave equations with damping and source terms. We prove that the solutions of the problem are unbounded when the initial data are large enough in some sense.

\section{Introduction}

In this work, we consider the following initial-boundary value problem:

$$
\begin{array}{r}
u_{t t}-\Delta u_{t}+\left|u_{t}\right|^{p-1} u_{t}=\operatorname{div}\left(\rho\left(|\nabla u|^{2}\right) \nabla u\right)+f_{1}(u, v), \\
(x, t) \in \Omega \times(0, T), \\
v_{t t}-\Delta v_{t}+\left|v_{t}\right|^{q-1} v_{t}=\operatorname{div}\left(\rho\left(|\nabla v|^{2}\right) \nabla v\right)+f_{2}(u, v), \\
(x, t) \in \Omega \times(0, T), \\
u(x, t)=v(x, t)=0, \quad(x, t) \in \partial \Omega \times(0, T), \\
u(x, 0)=u_{0}(x), \quad u_{t}(x, 0)=u_{1}(x), \quad x \in \Omega, \\
v(x, 0)=v_{0}(x), \quad v_{t}(x, 0)=v_{1}(x), \quad x \in \Omega,
\end{array}
$$

where $\Omega$ is a bounded domain with smooth boundary $\partial \Omega$ in $R^{n}, n=1,2,3 ; p, q \geq 1 ; f_{i}: R^{2} \rightarrow R$ are given functions to be specified later.

Throughout this paper, we define $\rho$ by

$$
\rho(s)=b_{1}+b_{2} s^{m}, \quad s \geq 0,
$$

where $b_{1}, b_{2}$ are nonnegative constants and $b_{1}+b_{2}>0$.

This type of problems not only is important from the theoretical point of view, but also arises in material science and physics that deal with system of nonlinear wave equations.
Ye [1] obtained the local existence and the blowup of the solution of problem (1), for $\rho(s)=s^{m}$. In the absence of the strong damping $\left(\Delta u_{t}\right.$ and $\left.\Delta v_{t}\right)$ terms, problem (1) becomes

$$
\begin{aligned}
& u_{t t}+\left|u_{t}\right|^{p-1} u_{t}=\operatorname{div}\left(\rho\left(|\nabla u|^{2}\right) \nabla u\right)+f_{1}(u, v), \\
& v_{t t}+\left|v_{t}\right|^{q-1} v_{t}=\operatorname{div}\left(\rho\left(|\nabla v|^{2}\right) \nabla v\right)+f_{2}(u, v) .
\end{aligned}
$$

Wu et al. [2] obtained the global existence and blowup of the solution of problem (3) under some suitable conditions. Fei and Hongjun [3] considered problem (3) and improved the blowup result obtained in [2], for a large class of initial data in positive initial energy, using the same techniques as in Payne and Sattinger [4] and some estimates used firstly by Vitillaro [5]. Recently, Pişkin and Polat $[6,7]$ studied the local and global existence, energy decay, and blowup of the solution of problem (3). Also, for more information about (1) and (3), see $[2,3,7]$.

The many problems associated with (1) are studied from various aspects in many papers [8-13].

In this work, we will consider the blowup property in infinity time, that is, exponential growth.

This work is organized as follows. In Section 2, we state the local existence result. In Section 3, we establish that the energy will grow up as an exponential as time goes to infinity, provided that the initial data are large enough or $E(0)<E_{1}$, where $E(0)$ and $E_{1}$ are defined in (9) and (15). 


\section{Preliminaries}

In this section, we introduce some notations and lemmas and local existence theorem needed in the proof of our main results. Let $\|\cdot\|$ and $\|\cdot\|_{p}$ denote the usual $L^{2}(\Omega)$ norm and $L^{p}(\Omega)$ norm, respectively.

Concerning the functions $f_{1}(u, v)$ and $f_{2}(u, v)$, we take

$$
\begin{aligned}
& f_{1}(u, v)=a|u+v|^{2(r+1)}(u+v)+b|u|^{r} u|v|^{r+2} \\
& f_{2}(u, v)=a|u+v|^{2(r+1)}(u+v)+b|v|^{r} v|u|^{r+2}
\end{aligned}
$$

where $a, b>0$ are constants and $r$ satisfies

$$
\begin{gathered}
-1<r \quad \text { if } n \leq 2, \\
-1<r \leq 1 \quad \text { if } n=3 .
\end{gathered}
$$

According to the above equalities they can easily verify that

$$
u f_{1}(u, v)+v f_{2}(u, v)=2(r+2) F(u, v), \quad \forall(u, v) \in R^{2}
$$

where

$$
F(u, v)=\frac{1}{2(r+2)}\left[a|u+v|^{2(r+2)}+2 b|u v|^{r+2}\right]
$$

We have the following result.

Lemma 1 (see [14]). There exist two positive constants $c_{0}$ and $c_{1}$ such that

$$
\begin{aligned}
c_{0}\left(|u|^{2(r+2)}+|v|^{2(r+2)}\right) & \leq 2(r+2) F(u, v) \\
& \leq c_{1}\left(|u|^{2(r+2)}+|v|^{2(r+2)}\right)
\end{aligned}
$$

is satisfied.

We define the energy function as follows:

$$
\begin{aligned}
E(t)= & \frac{1}{2}\left(\left\|u_{t}\right\|^{2}+\left\|v_{t}\right\|^{2}\right) \\
& +\frac{1}{2} \int_{\Omega}\left(P\left(|\nabla u|^{2}\right)+P\left(|\nabla v|^{2}\right)\right) d x \\
& -\int_{\Omega} F(u, v) d x,
\end{aligned}
$$

where $P(s)=\int_{0}^{s} \rho(\xi) d \xi, s \geq 0$.

Lemma 2 (see [7]). E(t) is a nonincreasing function for $t \geq 0$ and

$$
E^{\prime}(t)=-\left(\left\|\nabla u_{t}\right\|^{2}+\left\|\nabla v_{t}\right\|^{2}+\left\|u_{t}\right\|_{p+1}^{p+1}+\left\|v_{t}\right\|_{q+1}^{q+1}\right) \leq 0 .
$$

Lemma 3 (Sobolev-Poincare inequality [15]). Let $p$ be a number with $2 \leq p<\infty(n=1,2)$ or $2 \leq p \leq 2 n /(n-2)(n \geq$ $3)$, then there is a constant $C_{*}=C_{*}(\Omega, p)$ such that

$$
\|u\|_{p} \leq C_{*}\|\nabla u\| \quad \text { for } u \in H_{0}^{1}(\Omega)
$$

Next, we state the local existence theorem $[1,7]$.

Theorem 4 (local existence). Suppose that (5) holds. Then there exist $p, q$ satisfying

$$
\begin{gathered}
1 \leq p, q \quad \text { if } n \leq 2, \\
1 \leq p, q \leq 5 \text { if } n=3,
\end{gathered}
$$

and further $\left(u_{0}, v_{0}\right) \in\left(W_{0}^{1,2(m+1)}(\Omega) \cap L^{2(r+2)}(\Omega)\right) \times$ $\left(W_{0}^{1,2(m+1)}(\Omega) \cap L^{2(r+2)}(\Omega)\right),\left(u_{1}, v_{1}\right) \in L^{2}(\Omega) \times L^{2}(\Omega)$. Then problem (1) has a unique local solution

$$
\begin{gathered}
u, v \in C\left([0, T) ; W_{0}^{1,2(m+1)}(\Omega) \cap L^{2(r+2)}(\Omega)\right), \\
u_{t} \in C\left([0, T) ; L^{2}(\Omega)\right) \cap L^{p+1}(\Omega \times[0, T)), \\
v_{t} \in C\left([0, T) ; L^{2}(\Omega)\right) \cap L^{q+1}(\Omega \times[0, T)) .
\end{gathered}
$$

\section{Exponential Growth}

In this section, we will prove that the energy is unbounded when the initial data are large enough in some sense. Our techniques of proof follow very carefully the techniques used in [16].

Lemma 5 (see [3]). Suppose that (5) holds. Then there exists $\eta>0$ such that for any $(u, v) \in\left(W_{0}^{1,2(m+1)}(\Omega) \cap L^{2(r+2)}(\Omega)\right) \times$ $\left(W_{0}^{1,2(m+1)}(\Omega) \cap L^{2(r+2)}(\Omega)\right)$ the inequality

$$
\begin{aligned}
\| u & +v\left\|_{2(r+2)}^{2(r+2)}+2\right\| u v \|_{r+2}^{r+2} \\
& \leq \eta\left(\int_{\Omega}\left(P\left(|\nabla u|^{2}\right)+P\left(|\nabla v|^{2}\right)\right) d x\right)^{r+2}
\end{aligned}
$$

holds.

For the sake of simplicity and to prove our result, we take $a=b=1$ and introduce

$$
\begin{gathered}
B=\eta^{1 / 2(r+2)}, \quad \alpha_{1}=B^{-((r+2) /(r+1))}, \\
E_{1}=\left(\frac{1}{2}-\frac{1}{2(r+2)}\right) \alpha_{1}^{2},
\end{gathered}
$$

where $\eta$ is the optimal constant in (14). Next, we will state a lemma which is similar to the one introduced firstly by Vitillaro in [5] to study a class of a single wave equation.

Lemma 6 (see [3]). Suppose that (5) holds. Let $(u, v)$ be the solution of problem (1). Assume further that $E(0)<E_{1}$ and

$$
\left(\int_{\Omega}\left(P\left(\left|\nabla u_{0}\right|^{2}\right)+P\left(\left|\nabla v_{0}\right|^{2}\right)\right) d x\right)^{1 / 2}>\alpha_{1} .
$$


Then there exists a constant $\alpha_{2}>\alpha_{1}$ such that

$$
\begin{aligned}
& \left(\int_{\Omega}\left(P\left(|\nabla u|^{2}\right)+P\left(|\nabla v|^{2}\right)\right) d x\right)^{1 / 2} \geq \alpha_{2} \\
& \left(\|u+v\|_{2(r+2)}^{2(r+2)}+2\|u v\|_{r+2}^{r+2}\right)^{1 / 2(r+2)} \geq B \alpha_{2}
\end{aligned}
$$

for all $t \in[0, T)$.

Theorem 7. Suppose that (5) and $2(r+2)>\max \{p+1, q+$ $1\}$ hold. Then any solution of problem (1) with initial data satisfying

$$
\left(\int_{\Omega}\left(P\left(\left|\nabla u_{0}\right|^{2}\right)+P\left(\left|\nabla v_{0}\right|^{2}\right)\right) d x\right)^{1 / 2}>\alpha_{1}, \quad E(0)<E_{1}
$$

grows exponentially.

Proof. We set

$$
H(t)=E_{1}-E(t), \quad t \geq 0 .
$$

From (10) and (20) we get

$$
H^{\prime}(t)=-E^{\prime}(t)=\left\|u_{t}\right\|_{p+1}^{p+1}+\left\|v_{t}\right\|_{q+1}^{q+1} \geq 0 ;
$$

hence we have $H(t) \geq H(0)=E_{1}-E(0)>0$.

We consider the following functional:

$$
L(t)=H(t)+\varepsilon \int_{\Omega}\left(u u_{t}+v v_{t}\right) d x+\frac{\varepsilon}{2}\left(\|\nabla u\|^{2}+\|\nabla v\|^{2}\right)
$$

for small $\varepsilon$ to be specified later.

Our goal is to show that $L(t)$ satisfies a differential inequality of the form

$$
L^{\prime}(t) \geq C L(t) \quad \forall t \geq 0 .
$$

This, of course, will lead to exponential growth.

By taking a derivative of (22) and using (1), it follows that

$$
\begin{aligned}
L^{\prime}(t)= & H^{\prime}(t)+\varepsilon\left(\left\|u_{t}\right\|^{2}+\left\|v_{t}\right\|^{2}\right) \\
& +\varepsilon \int_{\Omega}\left(u u_{t t}+v v_{t t}\right) d x \\
& +\varepsilon \int_{\Omega}\left(\nabla u \nabla u_{t}+\nabla v \nabla v_{t}\right) d x \\
= & \left(\left\|u_{t}\right\|_{p+1}^{p+1}+\left\|v_{t}\right\|_{q+1}^{q+1}\right)+\varepsilon\left(\left\|u_{t}\right\|^{2}+\left\|v_{t}\right\|^{2}\right) \\
& -\varepsilon b_{1}\left(\|\nabla u\|^{2}+\|\nabla v\|^{2}\right) \\
& -\varepsilon b_{2}\left(\|\nabla u\|_{2(m+1)}^{2(m+1)}+\|\nabla v\|_{2(m+1)}^{2(m+1)}\right) \\
& -\varepsilon \int_{\Omega}\left(u f_{1}(u, v)+v f_{2}(u, v)\right) d x \\
& -\varepsilon \int_{\Omega}\left(u\left|u_{t}\right|^{p-1} u_{t}+v\left|v_{t}\right|^{q-1} v_{t}\right) d x .
\end{aligned}
$$

From (9) and (20), it follows that

$$
\begin{aligned}
-b_{2}( & \left(\|\nabla u\|_{2(m+1)}^{2(m+1)}+\|\nabla v\|_{2(m+1)}^{2(m+1)}\right) \\
= & 2(m+1) H(t)-2(m+1) E_{1} \\
& +(m+1)\left(\left\|u_{t}\right\|^{2}+\left\|v_{t}\right\|^{2}\right) \\
& +b_{1}(m+1)\left(\|\nabla u\|^{2}+\|\nabla v\|^{2}\right) \\
& -2(m+1) \int_{\Omega} F(u, v) d x .
\end{aligned}
$$

Inserting (25) into (24), we get

$$
\begin{aligned}
L^{\prime}(t)= & \left(\left\|u_{t}\right\|_{p+1}^{p+1}+\left\|v_{t}\right\|_{q+1}^{q+1}\right) \\
& +\varepsilon(m+2)\left(\left\|u_{t}\right\|^{2}+\left\|v_{t}\right\|^{2}\right) \\
& +\varepsilon b_{1} m\left(\|\nabla u\|^{2}+\|\nabla v\|^{2}\right) \\
& +\varepsilon\left(1-\frac{m+1}{r+2}\right)\left(\|u+v\|_{2(r+2)}^{2(r+2)}+2\|u v\|_{r+2}^{r+2}\right) \\
& +2 \varepsilon(m+1) H(t)-2 \varepsilon(m+1) E_{1} \\
& -\varepsilon \int_{\Omega}\left(u\left|u_{t}\right|^{p-1} u_{t}+v\left|v_{t}\right|^{q-1} v_{t}\right) d x .
\end{aligned}
$$

Then using (18), we obtain

$$
\begin{aligned}
L^{\prime}(t) \geq & \left(\left\|u_{t}\right\|_{p+1}^{p+1}+\left\|v_{t}\right\|_{q+1}^{q+1}\right) \\
& +\varepsilon(m+2)\left(\left\|u_{t}\right\|^{2}+\left\|v_{t}\right\|^{2}\right) \\
& +\varepsilon b_{1} m\left(\|\nabla u\|^{2}+\|\nabla v\|^{2}\right)+2 \varepsilon(m+1) H(t) \\
& +\varepsilon c^{\prime}\left(\|u+v\|_{2(r+2)}^{2(r+2)}+2\|u v\|_{r+2}^{r+2}\right) \\
& -\varepsilon \int_{\Omega}\left(u\left|u_{t}\right|^{p-1} u_{t}+v\left|v_{t}\right|^{q-1} v_{t}\right) d x
\end{aligned}
$$

where $c^{\prime}=1-(m+1) /(r+2)-2(m+1) E_{1}\left(B \alpha_{2}\right)^{-2(r+2)}$. It is clear that $c^{\prime}>0$, since $\alpha_{2}>B^{-((r+2) /(r+1))}$. In order to estimate the last two terms in (27), we use the following Young inequality:

$$
X Y \leq \frac{\delta^{k} X^{k}}{k}+\frac{\delta^{-l} Y^{l}}{l}
$$

where $X, Y \geq 0, \delta>0, k, l \in R^{+}$such that $1 / k+1 / l=1$. Consequently, applying the above inequality we have

$$
\begin{gathered}
\int_{\Omega} u u_{t}\left|u_{t}\right|^{p-1} d x \leq \frac{\delta_{1}^{p+1}}{p+1}\|u\|_{p+1}^{p+1}+\frac{p \delta_{1}^{-((p+1) / p)}}{p+1}\left\|u_{t}\right\|_{p+1}^{p+1}, \\
\int_{\Omega} v v_{t}\left|v_{t}\right|^{q-1} d x \leq \frac{\delta_{2}^{q+1}}{q+1}\|v\|_{q+1}^{q+1}+\frac{q \delta_{2}^{-((q+1) / q)}}{q+1}\left\|v_{t}\right\|_{q+1}^{q+1} .
\end{gathered}
$$


Inserting estimates (29) into (27), we have

$$
\begin{aligned}
L^{\prime}(t) \geq & \varepsilon(m+2)\left(\left\|u_{t}\right\|^{2}+\left\|v_{t}\right\|^{2}\right) \\
& +\varepsilon b_{1} m\left(\|\nabla u\|^{2}+\|\nabla v\|^{2}\right)+2 \varepsilon(m+1) H(t) \\
& +\varepsilon c_{2}\left(\|u\|_{2(r+2)}^{2(r+2)}+\|v\|_{2(r+2)}^{2(r+2)}\right) \\
& -\varepsilon \frac{\delta_{1}^{p+1}}{p+1}\|u\|_{p+1}^{p+1}+\left(1-\varepsilon \frac{p \delta_{1}^{-((p+1) / p)}}{p+1}\right)\left\|u_{t}\right\|_{p+1}^{p+1} \\
& -\varepsilon \frac{\delta_{2}^{q+1}}{q+1}\|v\|_{q+1}^{q+1}+\left(1-\varepsilon \frac{q \delta_{2}^{-((q+1) / q)}}{q+1}\right)\left\|v_{t}\right\|_{q+1}^{q+1},
\end{aligned}
$$

where $c_{2}=c^{\prime} c_{0}$.

Since $2(r+2)>\max \{p+1, q+1\}$, from the embedding $L^{2(r+2)}(\Omega) \hookrightarrow L^{p+1}(\Omega)$ and embedding $L^{2(r+2)}(\Omega) \hookrightarrow$ $L^{p+1}(\Omega)$, we have

$$
\begin{gathered}
\|u\|_{p+1}^{p+1} \leq c_{3}\|u\|_{2(r+2)}^{p+1}, \\
\|v\|_{q+1}^{q+1} \leq c_{4}\|v\|_{2(r+2)}^{q+1}
\end{gathered}
$$

for some positive constants $c_{3}$ and $c_{4}$. Using the algebraic inequality

$$
\begin{aligned}
& z^{v} \leq z+1 \leq\left(1+\frac{1}{a}\right)(z+a), \\
& \forall z \geq 0, \quad 0<v \leq 1, \quad a \geq 0,
\end{aligned}
$$

and since $H(t) \geq H(0)$, we get

$$
\begin{aligned}
\|u\|_{2(r+2)}^{p+1} & \leq d\left(\|u\|_{2(r+2)}^{2(r+2)}+H(0)\right) \\
& \leq d\left(\|u\|_{2(r+2)}^{2(r+2)}+H(t)\right),
\end{aligned}
$$

where $d=1+1 / H(0)$. Similarly

$$
\|v\|_{2(r+2)}^{q+1} \leq d\left(\|v\|_{2(r+2)}^{2(r+2)}+H(t)\right) .
$$

Inserting (33) and (34) into (30), we have

$$
\begin{aligned}
L^{\prime}(t) \geq & \varepsilon(m+2)\left(\left\|u_{t}\right\|^{2}+\left\|v_{t}\right\|^{2}\right) \\
& +\varepsilon b_{1} m\left(\|\nabla u\|^{2}+\|\nabla v\|^{2}\right) \\
& +\varepsilon\left(2(m+1)-d \frac{\delta_{1}^{p+1}}{p+1}-d \frac{\delta_{2}^{q+1}}{q+1}\right) H(t) \\
& +\varepsilon\left(c_{2}-d \frac{\delta_{1}^{p+1}}{p+1}-d \frac{\delta_{2}^{q+1}}{q+1}\right)\left(\|u\|_{2(r+2)}^{2(r+2)}+\|v\|_{2(r+2)}^{2(r+2)}\right) \\
& +\left(1-\varepsilon \frac{p \delta_{1}^{-((p+1) / p)}}{p+1}\right)\left\|u_{t}\right\|_{p+1}^{p+1} \\
& +\left(1-\varepsilon \frac{q \delta_{2}^{-((q+1) / q)}}{q+1}\right)\left\|v_{t}\right\|_{q+1}^{q+1} .
\end{aligned}
$$

Now, once $\delta_{1}$ and $\delta_{2}$ are fixed, we can choose $\varepsilon$ small enough such that

$$
\begin{gathered}
1-\varepsilon \frac{p \delta_{1}^{-((p+1) / p)}}{p+1}>0, \quad 1-\varepsilon \frac{q \delta_{2}^{-((q+1) / q)}}{q+1}>0, \\
L(0)=H(0)+\varepsilon \int_{\Omega}\left(u_{0} u_{1}+v_{0} v_{1}\right) d x>0 .
\end{gathered}
$$

Consequently (35) takes the form

$$
\begin{aligned}
L^{\prime}(t) \geq & \varepsilon(m+2)\left(\left\|u_{t}\right\|^{2}+\left\|v_{t}\right\|^{2}\right) \\
& +\varepsilon b_{1} m\left(\|\nabla u\|^{2}+\|\nabla v\|^{2}\right) \\
& +\varepsilon\left(2(m+1)-d \frac{\delta_{1}^{p+1}}{p+1}-d \frac{\delta_{2}^{q+1}}{q+1}\right) H(t) \\
& +\varepsilon\left(c_{2}-d \frac{\delta_{1}^{p+1}}{p+1}-d \frac{\delta_{2}^{q+1}}{q+1}\right)\left(\|u\|_{2(r+2)}^{2(r+2)}+\|v\|_{2(r+2)}^{2(r+2)}\right) \\
\geq & \theta\left(\left\|u_{t}\right\|^{2}+\left\|v_{t}\right\|^{2}+H(t)+\|\nabla u\|^{2}\right. \\
& \left.+\|\nabla v\|^{2}+\|u\|_{2(r+2)}^{2(r+2)}+\|v\|_{2(r+2)}^{2(r+2)}\right),
\end{aligned}
$$

where $\theta=\min \left\{\varepsilon(m+2), \varepsilon b_{1} m,\left(2(m+1)-d\left(\delta_{1}^{p+1} /(p+1)\right)-\right.\right.$ $\left.\left.d\left(\delta_{2}^{q+1} /(q+1)\right)\right), \varepsilon\left(c_{2}-d\left(\delta_{1}^{p+1} /(p+1)\right)-d\left(\delta_{2}^{q+1} /(q+1)\right)\right)\right\}$.

Then we have

$$
L(t) \geq L(0)>0, \quad \forall t \geq 0 .
$$

On the other hand, applying Hölder inequality, we obtain

$$
\begin{aligned}
& \left|\int_{\Omega} u u_{t} d x+\int_{\Omega} v v_{t} d x\right| \\
& \quad \leq\|u\|\left\|u_{t}\right\|+\|v\|\left\|v_{t}\right\| \\
& \quad \leq C\left(\|u\|_{2(r+2)}\left\|u_{t}\right\|+\|v\|_{2(r+2)}\left\|v_{t}\right\|\right) .
\end{aligned}
$$

Young inequality gives

$$
\begin{gathered}
\left|\int_{\Omega} u u_{t} d x+\int_{\Omega} v v_{t} d x\right| \\
\leq \frac{C}{2}\left(\|u\|_{2(r+2)}^{2}+\left\|u_{t}\right\|^{2}+\|v\|_{2(r+2)}^{2}+\left\|v_{t}\right\|^{2}\right) \\
\leq \frac{C}{2}\left(\left(\|u\|_{2(r+2)}^{2(r+2)}\right)^{1 /(r+2)}+\left\|u_{t}\right\|^{2}\right. \\
\left.+\left(\|v\|_{2(r+2)}^{2(r+2)}\right)^{1 /(r+2)}+\left\|v_{t}\right\|^{2}\right) .
\end{gathered}
$$

Since $r>-1$, algebraic inequality (32) yields

$$
\begin{aligned}
& \left|\int_{\Omega} u u_{t} d x+\int_{\Omega} v v_{t} d x\right| \\
& \leq \frac{C}{2}\left(\left(1+\frac{1}{H(0)}\right)\left(\|u\|_{2(r+2)}^{2(r+2)}+H(t)\right)+\left\|u_{t}\right\|^{2}\right. \\
& \left.\quad+\left(1+\frac{1}{H(0)}\right)\left(\|v\|_{2(r+2)}^{2(r+2)}+H(t)\right)+\left\|v_{t}\right\|^{2}\right) .
\end{aligned}
$$


Note that

$$
\begin{aligned}
L(t) & \leq H(t)+\varepsilon \int_{\Omega}\left(u u_{t}+v v_{t}\right) d x \\
& \leq H(t)+\left|\int_{\Omega} u u_{t} d x+\int_{\Omega} v v_{t} d x\right| \\
& \leq C^{\prime}\left(\left\|u_{t}\right\|^{2}+\left\|v_{t}\right\|^{2}+H(t)+\|\nabla u\|^{2}+\|\nabla v\|^{2}\right. \\
& \left.+\|u\|_{2(r+2)}^{2(r+2)}+\|v\|_{2(r+2)}^{2(r+2)}\right) .
\end{aligned}
$$

Combining with (37) and (42), we arrive at

$$
L^{\prime}(t) \geq C L(t) \quad \forall t \geq 0 .
$$

Integrating differential inequality (43) between 0 and $t$ gives the following estimate for $L(t)$ :

$$
L(t) \geq L(0) e^{C t}
$$

The proof of Theorem 7 is completed.

Remark 8. When $E(0)<0$, by setting $H(t)=-E(t)$, the similar result is obtained by applying the same arguments in the proof of Theorem 7 .

\section{Conflict of Interests}

The author declares that there is no conflict of interests regarding the publication of this paper.

\section{References}

[1] Y. Ye, "Existence and decay estimate of global solutions to systems of nonlinear wave equations with damping and source terms," Abstract and Applied Analysis, vol. 2013, Article ID 903625, 9 pages, 2013.

[2] J. Wu, S. Li, and S. Chai, "Existence and nonexistence of a global solution for coupled nonlinear wave equations with damping and source," Nonlinear Analysis: Theory, Methods \& Applications, vol. 72, no. 11, pp. 3969-3975, 2010.

[3] L. Fei and G. Hongjun, "Global nonexistence of positive initialenergy solutions for coupled nonlinear wave equations with damping and source terms," Abstract and Applied Analysis, vol. 2011, Article ID 760209, 14 pages, 2011.

[4] L. E. Payne and D. H. Sattinger, "Saddle points and instability of nonlinear hyperbolic equations," Israel Journal of Mathematics, vol. 22, no. 3-4, pp. 273-303, 1975.

[5] E. Vitillaro, "Global nonexistence theorems for a class of evolution equations with dissipation," Archive for Rational Mechanics and Analysis, vol. 149, no. 2, pp. 155-182, 1999.

[6] E. Pişkin, "Blow up for coupled nonlinear wave equations with weak damping terms," International Journal of Differential Equations and Applications, vol. 12, no. 4, pp. 131-137, 2013.

[7] E. Pişkin and N. Polat, "Global existence, decay and blow up solutions for coupled nonlinear wave equations with damping and source terms," Turkish Journal of Mathematics, vol. 37, no. 4, pp. 633-651, 2013.

[8] W. Liu, "Arbitrary rate of decay for a viscoelastic equation with acoustic boundary conditions," Applied Mathematics Letters, vol. 38, pp. 155-161, 2014.
[9] W. Liu and S. Li, "General decay of solutions for a weak viscoelastic equation with acoustic boundary conditions," Zeitschrift für angewandte Mathematik und Physik, vol. 65, no. 1, pp. 125-134, 2014.

[10] Q. Bie and C. Luo, "Blow-up of solutions for a system of nonlinear viscoelastic wave equations with damping and source terms," Mathematica Applicata, vol. 24, no. 3, pp. 479-487, 2011.

[11] Y. Xiong, "Blow-up and polynomial decay of solutions for a viscoelastic equation with a nonlinear source," Zeitschrift für Analysis und ihre Anwendungen, vol. 31, no. 3, pp. 251-266, 2012.

[12] C. Wenying and X. Yangping, "Blow-up and general decay of solutions for a nonlinear viscoelastic equation," Electronic Journal of Differential Equations, no. 12, 11 pages, 2013.

[13] S.-T. Wu, "General decay and blow-up of solutions for a viscoelastic equation with nonlinear boundary dampingsource interactions," Zeitschrift für angewandte Mathematik und Physik, vol. 63, no. 1, pp. 65-106, 2012.

[14] S. A. Messaoudi and B. Said-Houari, "Global nonexistence of positive initial-energy solutions of a system of nonlinear viscoelastic wave equations with damping and source terms," Journal of Mathematical Analysis and Applications, vol. 365, no. 1, pp. 277-287, 2010.

[15] R. A. Adams and J. J. Fournier, Sobolev Spaces, vol. 140 of Pure and Applied Mathematics, Academic Press, 2nd edition, 2003.

[16] B. Said-Houari, "Exponential growth of positive initial-energy solutions of a system of nonlinear viscoelastic wave equations with damping and source terms," Zeitschrift für Angewandte Mathematik und Physik, vol. 62, no. 1, pp. 115-133, 2011. 


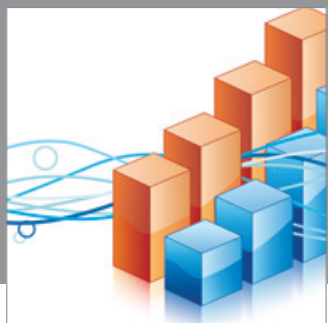

Advances in

Operations Research

mansans

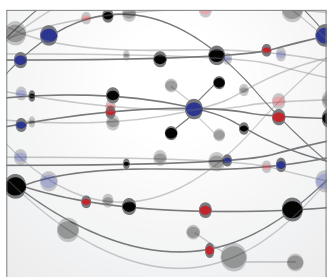

The Scientific World Journal
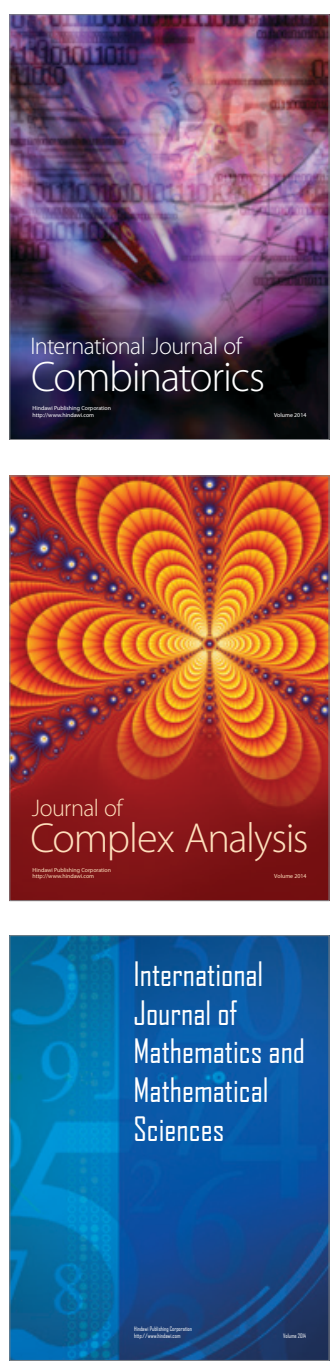
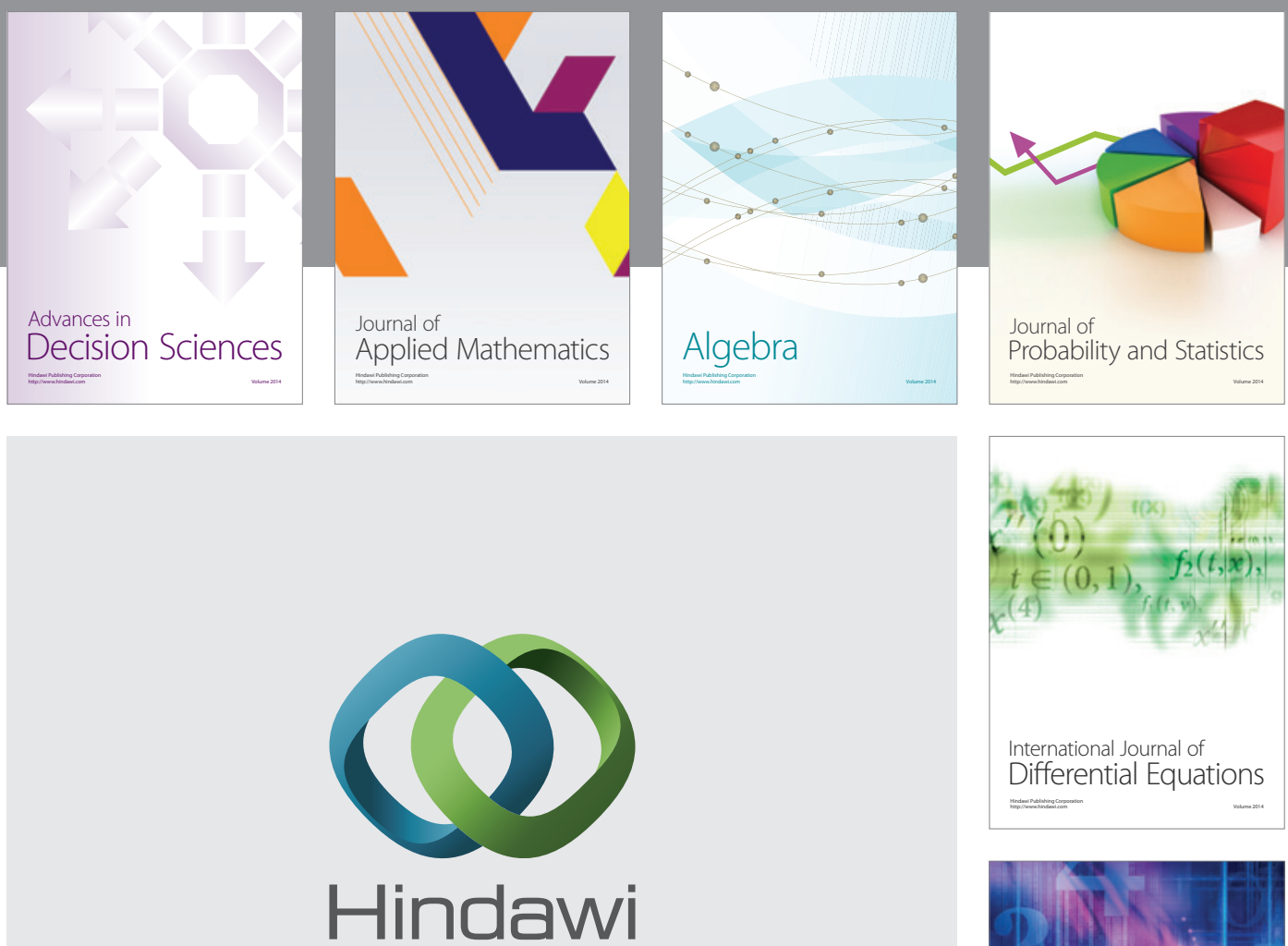

Submit your manuscripts at http://www.hindawi.com
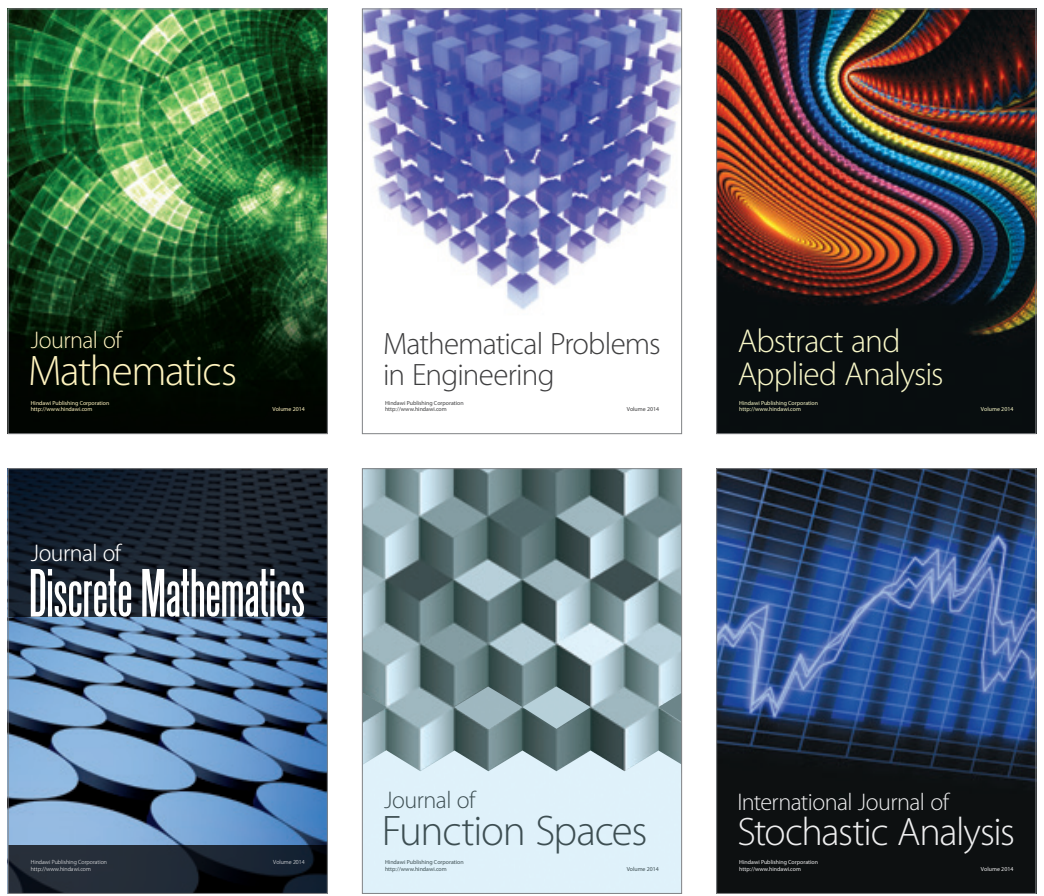

Journal of

Function Spaces

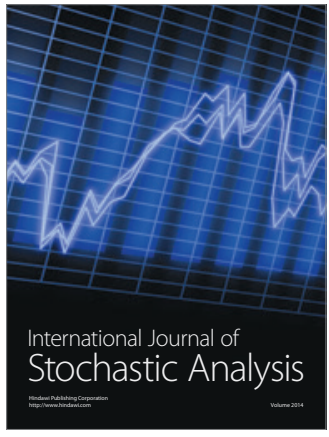

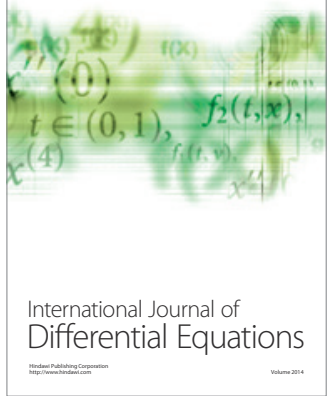
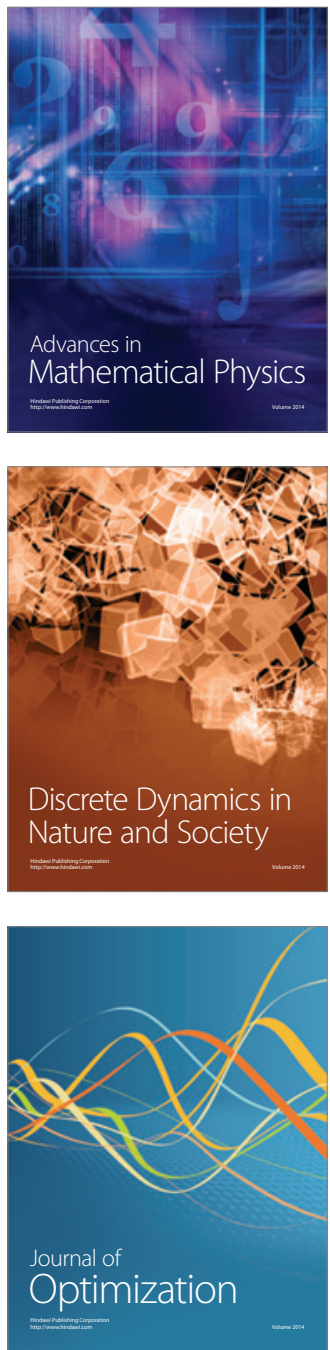